\title{
Preparation, analysis and antioxidant evaluation of the controlled product of polysaccharide from Mactra veneriformis \\ by mild acid hydrolysis
}

\author{
Ling Chong Wang ${ }^{\mathrm{a}, \mathrm{b} *}$, Hao $\mathrm{Wu}^{\mathrm{a}, \mathrm{b} *}$, Jing Ji ${ }^{\mathrm{c}}$, Feng Xue ${ }^{\mathrm{a}}$, Rui Liu ${ }^{\mathrm{a}, \mathrm{b}}$ \\ ${ }^{a}$ School of Pharmacy, Nanjing University of Chinese Medicine, Nanjing, P.R. China, 210023 \\ b Jiangsu Key Laboratory of Research and Development in Marine Bio-resource Pharmaceutics, \\ Nanjing University of Chinese Medicine Nanjing, P.R. China, 210023 \\ ${ }^{c}$ Center for drug safety evaluation and Research, Nanjing University of Chinese Medicine, P.R. \\ China, 210023
}

*Send Correspondence to: Associate Prof. Ling Chong Wang School of Pharmacy, Nanjing University of Chinese Medicine No. 138, Xianlin thoroughfare, Nanjing, P.R. China, 210029 Tel.: 86-025-85811207

E-mail address: 993wlc@njucm.edu.cn;

Or *send correspondence to: Prof. Hao Wu

School of Pharmacy, Nanjing University of Chinese Medicine

No. 138, Xianlin thoroughfare, Nanjing, P.R. China, 210029

Tel.: 86-025-85811206

E-mail address: whao5795@vip.sina.com 


\section{Abstract}

The polysaccharides from M. veneriformis (MVPS) were degraded by controlled mild acid hydrolysis to produce active oligosaccharides. MVPS can easily be hydrolyzed by $\mathrm{H}_{2} \mathrm{SO}_{4}$ or $\mathrm{HCl}$. The hydrolyzing process was investigated in acid addition and reaction time by evaluating the producing content of reducing sugar. Hydrolysis with $1 \mathrm{M} \mathrm{HCl}$ to MVPS can generate a time-depended behavior that is mild and controllable. HPLC analysis monitored the change of oligosaccharides composition in hydrolyzing. Total nine oligosaccharides are recognizable in the HPLC profile, and their content showed a regular transformation in hydrolysis. Those nine ingredients were identified as glucooligosaccharides with DP from 1 to 7 by MS analysis. Antioxidant activities of the typical hydrolyzates as well as MVPs were further tested in assays of DPPH and hydroxyl radicals scavenging, and reducing power. It was found that $\mathrm{HCl}$ hydrolyzate exhibited stronger antioxidant effects than MVPS and $\mathrm{H}_{2} \mathrm{SO}_{4}$ hydrolyzate due to its higher content of oligosaccharides.

Keywords: M. veneriformis; Polysaccharide; Hydrolyzation; Oligosaccharide; Antioxidant activity Chemical compounds studied in this article

Alpha-D-glucose (PubChem CID: 79025); Maltose (PubChem CID: 109991481); Kojibiose (PubChem CID: 164939); Maltotriose (PubChem CID: 109991481) 


\section{Introduction}

M. veneriformis, a typical marine bivalve mollusk, is a delicious sea food and a traditional Chinese medicine, while is low-cost, ubiquitous and abundant in Chinese coastal areas, especially in the coastal shoals of Jiangsu province. This marine clam was found containing high content of polysaccharide (Kasai, Horie, \& Sakamoto, 2004; Luan, Wang, Wu, Jin, \& Ji, 2011). By boiling its flesh in hot water and precipitating the decoction with ethanol, a crude polysaccharide extract with about $3.2 \%$ of yield can be acquired (Wang, Zhang, Di, Liu, \& Wu, 2011). The crude polysaccharide extract has about $25 \%$ of protein impurities, which can be dislodged by trichloroacetic acid (TCA) disposal. After deproteinization, the crude polysaccharide was refined to pure M. veneriformis polysaccharide (MVPS) with total sugar content higher than 95\% and protein content lower than 1.0\%. At least three homogeneous polysaccharides, named MVPS-1, MVPS-2 and MVPS-3, can be isolated from MVPS by DEAE-52 column separation and all of them showed about $450 \mathrm{kDa}$ of average molecular mass in GPC analysis. However, both MVPS and its three subdivided ingredients contain only D-glucose in monosaccharide composition. The D-glucose units in polysaccharide are linked each other by $\alpha-(1 \rightarrow 4)$ or $\alpha-(1 \rightarrow 2)$ glycosidic bonds and they appeared in the molecule with 6:1 of mole ratio. The sequence structures of MVPS are linear and no branches formation, in which six $\alpha-(1 \rightarrow 4)$ linked glucoses connected with one $\alpha-(1 \rightarrow 2)$ linked glucose are continuously repeated. Thus, the special molecular chain structure of MVPS can be vividly expressed with $[\rightarrow 4 \mathrm{Glc} 1 \rightarrow 4 \mathrm{Glc} 1 \rightarrow 4 \mathrm{Glc} 1 \rightarrow 2 \mathrm{Glc} 1 \rightarrow 4 \mathrm{Glc} 1 \rightarrow 4 \mathrm{Glc} 1 \rightarrow 4 \mathrm{Glc} 1]_{\mathrm{n}}$.

Additionally, MVPS has been proved having huge exploring potentials as functional foods and nutritional supplements. Early studies demonstrated that MVPS has hyperglycemia inhibiting, immunomodulatory, hepatic protecting and antioxidant activities in vitro or in vivo (Wang, Wu, Chang, \& Zhang, 2011; Zheng, Wang, Wu, \& Luan, 2011). Animal experimental tests showed that the crude and refining MVPS exhibited properly activities in glycemia inhibition and 300 $\mathrm{mg} / \mathrm{kg}$-weight has the optimal effect on hyperglycemia ICR mice that induced by alloxan injection. Oral administration of MVPS can increase the relative spleen and thymus weight of immunodeficiency rat, promote secretion of tumor necrosis factor alpha (TNF- $\alpha$ ), stimulate lymphocyte proliferation, and augment phagocytosis and secretion of NO and TNF- $\alpha$ in peritoneal 
macrophages.

As far as the researches on natural active polysaccharide are concerned, structural modification is one important method to enhance or transform the activities of polysaccharide. Recent studies demonstrated that natural polysaccharides exhibiting their physiological functions by oral administration to animals are correlated to the oligosaccharide derivatives produced by gastric acid or enzymes in digestive tract (Beveridge, \& Richards, 1973; Agbagla-Dohnani, Cornu, \& Broudiscou, 2012). It is well known that treatment of polysaccharide with acid hydrolysis can interrupt the polymer chain, generating the less molecular mass of carbohydrates (oligosaccharides) (Du, Song, Hu, Liao, Ni, \& Li, 2011). The produced oligosaccharides exhibited improving physiological activities in contrast with their initial polysaccharides because they are easier to be absorbed into blood circulations and have higher bioavailability in organisms (Al Jamal, Roughley, \& Ludwig, 2001; Juskiewicz, Zdunczyk, Jankowski, Krol, \& Milala, 2008). Sometimes, the derived oligosaccharides might bring some novel biological effect due to their different structures and physicochemical properties when compared with their parent polysaccharide, such as chitooligosaccharide and chitosan (Aam, Heggset, Norberg, Sorlie, Varum, \& Eijsink, 2010), agaro-oligosaccharide and agar (Chen, Zheng, Lin, \& Yan, 2004), fructooligosaccharide and fructan (Apolinario, Goulart de Lima Damasceno, de Macedo Beltrao, Pessoa, Converti, \& da Silva, 2014), and so on (de Moura, Macagnan, \& da Silva, 2015). Those beneficial reports would support our study on the hydrolysis of MVPS. However, rare research had been performed on hydrolysis of MVPS and the bioactive degrading products never been reported before.

Therefore, MVPS was regularly processed with partial acid hydrolysis in this study. By this way, some active oligosaccharides might be desired at controlled hydrolysis extent by adjusting acid usage and hydrolyzing time. The active oligosaccharides form MVPS will be subsequently analyzed by HPLC and LC-MS like some previous reports (Chen, Zheng, Lin, \& Yan, 2004; Vismeh, Humpula, Chundawat, Balan, Dale, \& Jones, 2013; Barroso, Dijkstra, Geerts, Lagerwerf, van Veelen, \& de $\mathrm{Ru}, 2002)$. Meanwhile, the in vitro antioxidant activity of the oligosaccharide product compared with the initial polysaccharide was further investigated.

\section{Materials and method}

\subsection{Materials}


MVPS used as the initial polysaccharides in this study were prepared by our lab as described by previous report (Wang, Zhang, Di, Liu, \& Wu, 2011). It is opalescent powders with higher than 97.3\% of total sugar content that referenced as standard glucose and measured by anthrone-sulfuric acid method (Laurentin, \& Edwards, 2003).

The standard D-glucose, 3,5-dinitrosalicylic acid (DNS), sodium hydroxide (NaOH), concentrated hydrochloric acid $(\mathrm{HCl})$ and sulfuric acid $\left(\mathrm{H}_{2} \mathrm{SO}_{4}\right)$ were of analytical grade from Sinopharm (Shanghai,China). Chromatographic grade acetonitrile $\left(\mathrm{CH}_{3} \mathrm{CN}\right)$ and methanol $(\mathrm{MeOH})$ were obtained from Thermo Fisher Scientific (Waltham, USA). MilliQ deionized water was used in this study. Ascorbic acid (Vc), 1,1-diphenyl-2-picrylhydrazyl (DPPH), safranin T, ferrous sulfate, potassium ferricyanide, hydrogen peroxide $\left(\mathrm{H}_{2} \mathrm{O}_{2}\right)$, ethylenediamine tetraacetic acid (EDTA) was purchased from Fluka Chemie (Buchs, Switzerland). Phosphate buffer saline with $\mathrm{pH}=7.4$ were purchased from Sigma Chemicals (St. Louis, USA).

\subsection{Mild acid hydrolysis of MVPS}

The hydrolysis action was investigated between two types of acid, $\mathrm{HCl}$ and $\mathrm{H}_{2} \mathrm{SO}_{4}$. Accordingly, four concentrations $(0.2,2,4$, and $6 \mathrm{M})$ of solution of $\mathrm{HCl}$ and $\mathrm{H}_{2} \mathrm{SO}_{4}$ were prepared as the reaction media. About 2.0 g MVPS was solubilized in $100 \mathrm{~mL}$ distilled water to prepared 20 $\mathrm{mg} / \mathrm{mL}$ stock solution. Every $10 \mathrm{~mL}$ stock solution was added into $10 \mathrm{~mL}$ of reaction media in one $50 \mathrm{~mL}$ conical flask under ice-bath condition. Then conical flasks were moved to water bath $\left(60^{\circ} \mathrm{C}\right)$ to start the acidic hydrolyzing reaction without agitation. During the reaction (from 0 to $300 \mathrm{~min}$ ), hydrolyzate $(200 \mu \mathrm{L})$ was withdrawn at set time and neutralized with $\mathrm{NaOH}$, then was measured its reducing sugar, or analyzed by HPLC and MS, or evaluated with antioxidant activities. The effect of acid usage on the hydrolysis was compared between $\mathrm{HCl}$ and $\mathrm{H}_{2} \mathrm{SO}_{4}$ at $0.1,1,2$, and $3 \mathrm{M}$ of concentration, which was analyzed through an Arrhenius-type plot of the kinetic behavior.

\subsection{Total and reducing sugars quantification}

The MVPS were quantified by measuring the reducing sugars after total acid hydrolysis, which were carried out in $1.0 \mathrm{~mL}$ reactions containing $10.0 \mathrm{mg}$ of MVPS and $6 \mathrm{M}$ of $\mathrm{HCl}$ at $60^{\circ}$ C for 12 h. Reducing sugars were quantified by the 3,5-dinitrosalicylic acid (DNS) method (Kai, Pengju, \& Guangye, 2008) using a standard curve of D-glucose. Hydrolysis degree was expressed as glucose equivalent (GE), defined as the percentage of total sugars converted into reducing sugars during the $\mathrm{HCl}$ or $\mathrm{H}_{2} \mathrm{SO}_{4}$ hydrolysis (Avila-Fernandez, Galicia-Lagunas, 
Rodriguez-Alegria, Olvera, \& Lopez-Munguia, 2011; Allerdings, Ralph, Steinhart, \& Bunzel, 2006).

\subsection{Composition analysis of oligosaccharides with HPLC-ELSD}

The oligomers analysis of the MVPS hydrolyzate was performed by high performance liquid chromatography (HPLC) with a method adapted from Chen, Zheng, Lin, \& Yan (2004). HPLC analysis was performed on a Waters ${ }^{\mathrm{TM}}$ Alliance 2695 HPLC Separation Module (Waltham, MA, USA) equipped with a Shodex Asahipak $\mathrm{NH}_{2} \mathrm{P}-4 \mathrm{E}$ column $(4.6 \times 250 \mathrm{~mm})$ in combination with a Dionex PA-1 guard column $(4.0 \mathrm{~mm} \times 50 \mathrm{~mm})$. Elution was performed with a flow rate of 1.0 $\mathrm{mL} / \mathrm{min}$ and a column oven temperature of $30{ }^{\circ} \mathrm{C}$. The injection volume was set to $10 \mu \mathrm{L}$. The acetonitrile (A) and Millipore water (B) were used as eluents. The following elution profile was used: $0-5 \mathrm{~min}$, isocratic $25 \%(\mathrm{v} / \mathrm{v})$ of $\mathrm{B} ; 5-35 \mathrm{~min}$, linear gradient from $25 \%$ to $50 \%$ of $\mathrm{B}$. The $\mathrm{NH}_{2} \mathrm{P}-4 \mathrm{E}$ column (NP) was coupled to an evaporative light scattering detector (ELSD, Waters ${ }^{\mathrm{TM}}$ 2424). The optimized parameters of ELSD were set at sprayer heating mode ( $80 \%$ power, $40^{\circ} \mathrm{C}$ ) as $90{ }^{\circ} \mathrm{C}$ for drift tube temperature, $40 \mathrm{psi}$ for nitrogen gas flow pressure, and gains to 50 . The peak area from the HPLC profile was obtained using Empower software (Waters). A semi-quantitative estimation of oligosaccharides concentration was obtained through the total area of the carbohydrates eluted in the range of 8-28 min.

\subsection{Oligosaccharides identification with LC-ESI-TOF-MS}

Identification of the oligosaccharides in MVPS hydrolyzate was performed on a HPLC-ESI-TOF-MS system. The system consisted of two successive coupled apparatus, a Waters 2695 HPLC Separation Module equipped with a Shodex Asahipak $\mathrm{NH}_{2} \mathrm{P}-4 \mathrm{E}$ column $(4.6 \times 250$ $\mathrm{mm}$ ), and a Time-of-Flight Mass Spectrometer (QTOF-MS, Waters QTOF Premier) equipped with an electrospray source (run in). The HPLC separation was performed by the method of HPLC-ELSD analysis that mentioned above, i.e., $30{ }^{\circ} \mathrm{C}$ of column oven temperature, $10 \mu \mathrm{L}$ of injection volume, $1.0 \mathrm{~mL} / \mathrm{min}$ of flow rate and gradient eluent with acetonitrile (A)-water (B) $(25 \%$ of B held for 5 min and linear gradient elution for next 30 min from $25 \%$ to $50 \%$ B). After the HPLC separation, elutes were then introduced into the ESI-TOF-MS device for analysis at positive ionization mode $[\mathrm{M}+\mathrm{Na}]^{+}$. Mass spectrometric data was acquired in MS scan mode. Data acquisition and analysis were performed using Waters MassLynx (version 4.1). Mass spectrometer settings for MS analysis were as follows: capillary voltage $3.0 \mathrm{kV}$, cone voltage $45 \mathrm{~V}$, source 
temperature $120{ }^{\circ} \mathrm{C}$, desolvation temperature $280{ }^{\circ} \mathrm{C}$, collision energy $5.0 \mathrm{~V}$, scan range 80-2000 Da. Gas (N2) flow used for cone and desolvatation were, respectively, 50 and $350 \mathrm{~L} / \mathrm{h}$.

\subsection{The in vitro antioxidant activity assay}

MVPS and its two hydrolyzates were used as test samples in antioxidant assays. The two hydrolyzates were recorded as $\mathrm{HCl}$ hydrolyzate and $\mathrm{H}_{2} \mathrm{SO}_{4}$ hydtolyzate because they were obtained from processing with $1 \mathrm{~h}$ of $1 \mathrm{M} \mathrm{HCl}$ and $\mathrm{H}_{2} \mathrm{SO}_{4}$ hydrolysis, respectively. The withdrawn hydrolysates were wiped off the residual acids by $\mathrm{NaOH}$ neutralization before using, and then were tested their antioxidant activity by some conventional methods involving DPPH, hydroxyl radicals scavenging and reducing power assay.

For the DPPH radical scavenging tests, a reliable reaction system was used as the description of Braca, De Tommasi, Di Bari, Pizza, Politi, \& Morelli (2001). One milliliter of all test samples (Vc, MVPS, $\mathrm{HCl}$ and $\mathrm{H}_{2} \mathrm{SO}_{4}$ hydrolyzates) with different concentrations (from 10 to $2000 \mu \mathrm{g} / \mathrm{mL}$ ) was added to $3 \mathrm{~mL}$ of a $0.004 \%$ (wt./v) $\mathrm{MeOH}$ solution of DPPH. Absorbance at $517 \mathrm{~nm}$ was measured after $30 \mathrm{~min}$, and the percent scavenging activity was calculated by absorbance difference between control (only DPPH solution) and test sample (DPPH solution plus sample) take part in the absorbance of control (only DPPH solution).

Hydroxyl radical-scavenging activity of the test samples (Vc, MVPS, $\mathrm{HCl}$ and $\mathrm{H}_{2} \mathrm{SO}_{4}$ hydrolyzates) was determined according to the safranin $\mathrm{T}$ fading assay (Li et al., 2013). The reaction system contained $1 \mathrm{~mL}$ sodium phosphate buffer $(15 \mathrm{mM}, \mathrm{pH} 7.4), 1 \mathrm{~mL} 360 \mathrm{~g} / \mathrm{mL}$ safranin $\mathrm{T}, 0.5 \mathrm{~mL} 2 \mathrm{mM}$ EDTA-FeSO ${ }_{4}, 1 \mathrm{~mL} 3 \% \mathrm{H}_{2} \mathrm{O}_{2}$ and $1 \mathrm{~mL}$ tested samples solution (concentration ranged from 10 to $2000 \mu \mathrm{g} / \mathrm{mL}$ ). After incubation at $37^{\circ} \mathrm{C}$ for $30 \mathrm{~min}$, hydroxyl radical was detected by monitoring the absorbance at $520 \mathrm{~nm}$ against a blank. In the control, test sample was substituted with distill water and the $\mathrm{H}_{2} \mathrm{O}_{2}$ was substituted with sodium phosphate buffer. The hydroxyl radical-scavenging ratio was calculated as the proportion of the absorbance difference between control and sample in the absorbance of the control.

The reducing power of MVPS hydrolyzate was determined referring to the method (Yuan, Carrington, \& Walsh, 2005) with some modifications. The different concentrations of samples (from 100 to $1600 \mu \mathrm{g} / \mathrm{mL}$ ) $1 \mathrm{~mL}$ were mixed with phosphate buffer $(2.5 \mathrm{~mL}, 0.2 \mathrm{M}, \mathrm{pH} 6.6)$ and potassium ferricyanide $\left[\mathrm{K}_{3} \mathrm{Fe}(\mathrm{CN})_{6}\right](2.5 \mathrm{~mL}, 1 \%)$. The mixture was incubated at $50{ }^{\circ} \mathrm{C}$ for 20 min. A portion $(2.5 \mathrm{~mL})$ of TCA $(10 \%)$ was added to the mixture which was then centrifuged for 
$10 \mathrm{~min}$ at $3000 \mathrm{rpm}$. The upper layer of solution $(2.5 \mathrm{~mL})$ was mixed with water $(2.5 \mathrm{~mL})$ and $\mathrm{FeCl}_{3}(0.5 \mathrm{~mL}, 0.1 \%)$, and the absorbance was measured at $700 \mathrm{~nm}$.

\subsection{Statistical analysis}

All data were shown as mean \pm standard deviation (S.D.). Statistical differences between the experimental groups were determined by one way ANOVA, and differences were considered to be statistically significant if $P<0.05$.

\section{Result and Discussion}

\subsection{Acid hydrolysis of MVPS and the glucose equivalent (GE) index}

For our study, $\mathrm{HCl}$ and $\mathrm{H}_{2} \mathrm{SO}_{4}$ were used to hydrolyze MVPS in aqueous solution system, and the effects acids type and usage on degraded behavior were investigated in detail. The hydrolyzing isotherms were drawn by measuring the relative content of reducing sugar (expressed with GE) in hydrolyzate following with hydrolyzing time. The measured GE of hydrolyzate for both $\mathrm{HCl}$ and $\mathrm{H}_{2} \mathrm{SO}_{4}$ exhibited first increasing then stable trends when hydrolysis prolonged. The platform of GE can be used to evaluate the final hydrolyzing degree and the first time reaching platform can assess the hydrolyzing speed. In such experiments, some important results can be visualized from Fig. 1. Firstly, $\mathrm{HCl}$ as well as $\mathrm{H}_{2} \mathrm{SO}_{4}$ can effectively degrade the MVPS. MVPS are amorphous polysaccharides and linear polymers with flexible chain due to containing $\alpha$-type glycosidic linkages (Wang, Zhang, Di, Liu, \& Wu, 2011; Wang, Di, Liu, \& Wu, 2013), and can be easily dissolved in aqueous solvents. In aqueous media, the stretched molecular induce the sugar unit linkages contact well with catalyzer (acids), therefore, hydrolysis reaction is more easily happen. Secondly, increasing acid usage can accelerate degradation of MVPS. For example, MVPS disposed with $0.1,1.0,2.0$, and $3.0 \mathrm{M}$ of $\mathrm{HCl}$ hydrolysis for 300 min can result in 5.2, 40.8, 66.5, and $96.1 \%$ of GE producing, respectively. Similarly, such conclusion can also be resulted by observing the $\mathrm{H}_{2} \mathrm{SO}_{4}$ hydrolysis to MVPS. Thirdly, MVPS were more easily degraded by $\mathrm{H}_{2} \mathrm{SO}_{4}$ other than $\mathrm{HCl}$ in such hydrolyzing system $(P<0.05) . \mathrm{H}_{2} \mathrm{SO}_{4}$ disposal exhibited with the higher $\mathrm{GE}$ value and shorter time to $\mathrm{GE}$ platform than $\mathrm{HCl}$ disposal under same condition. Within 60 min (a very short term) of $\mathrm{H}_{2} \mathrm{SO}_{4}$ disposal (at $3 \mathrm{~mol} / \mathrm{L}$ of excitation), almost all resultants in MVPS hydrolyzate have become monosaccharides because the GE nearly reached $90 \%$. In contrast, $\mathrm{HCl}$ hydrolysis only arrived at $56.7 \%$ of GE level. This might be attributed that $\mathrm{H}_{2} \mathrm{SO}_{4}$ is a polybasic acid and possesses stronger acidity and oxidbillity than $\mathrm{HCl}$. However, from the viewpoint on 
efficient oligosaccharides production, $\mathrm{HCl}$ is more suitable to hydrolyze MVPS because the reaction is mild and its process is controllable. The optimal reaction time and acid usage will thus depend on the desired oligomer composition and yield. Finally, a suitable hydrolysis can be achieved from an initial GE of $5.8 \%$ up to a GE of $30.5 \%$ within 180 min by using $1 \mathrm{M}$ of $\mathrm{HCl}$ at $60{ }^{\circ} \mathrm{C}$ condition. The GE of $30.5 \%$ at $180 \mathrm{~min}$ is a vital point in kinetic curve for $1 \mathrm{M} \mathrm{HCl}$ hydrolysis of MVPS. Before this point, the hydrolysis basically keeps at a constant rate. From this point onwards, the rate decreases rapidly until maximum hydrolysis with platform GE of $40.8 \%$ is reached at $300 \mathrm{~min}$.

\subsection{Analysis of oligosaccharides produced during the acid hydrolysis of MVPS}

Although the acid hydrolysis of MVPS has been roughly described in the above section, the evolution on the composition and distribution of carbohydrate profile for hydrolysis products has not yet been deeply considered. The carbohydrate profile of hydrolyzate at different hydrolysis time was obtained by high-performance chromatography with an evaporative light scattering detection (HPLC-ELSD). HPLC-ELSD has been widely used for the analysis of oligosaccharide because it allows the observation of short and longer linear oligosaccharide up to a degree of polymerization (DP) of about 30 . This technique has been previously applied to describe the carbohydrate profile of hydrolysis products of other polysaccharides (Arrizon, Morel, Gschaedler, \& Monsan, 2010). Thereby, it is also used to analysis the distribution of short oligosaccharide of MVPS hydrolyzate.

As shown in Fig. 2, fingerprint composed of serial separate eluting peaks in the chromatogram from retention time 8 to 35 min expressed the carbohydrate profile of the reaction liquors prescribed with different acid hydrolysis for suitable periods. Each component peak is appointed to a specific low-molecular-weight oligosaccharide with different DP. Theoretically, peaks eluted later through the separation column are oligosaccharides with higher DP than that eluted early (Chen, Zheng, Lin, \& Yan, 2004). Total nine eluting peaks from $\mathrm{HCl}$ hydrolyzates and seven eluting peaks from $\mathrm{H}_{2} \mathrm{SO}_{4}$ hydrolyzates are recognizable in the carbohydrate profile. These notable peaks are marked with consecutive numbers (1-9) according to their retention time. However, there are no available standards for their identification. It can be seen from Fig. 2 that the long chain molecules of MVPS are rapidly hydrolyzed to low molecule weight oligomer as the acidic hydrolysis proceeds. With regard to the $\mathrm{H}_{2} \mathrm{SO}_{4}$ hydrolysis, peaks $1,2,4,6,7$ are detected in 
hydrolyzate withdrawn at $0.5 \mathrm{~h}$; peaks 3 and 5 started to appear while peaks 6 and 7 are rapidly decayed at $1 \mathrm{~h}$; after that, all compounds peaks except peak 1 in $\mathrm{H}_{2} \mathrm{SO}_{4}$ hydrolyzate are mainly disappeared, indicating that oligosaccharides with higher DP were degraded into monose. However, in $\mathrm{HCl}$ hydrolyzate, peaks 2, 4, 6-9 obviously decreased (even to undetectable extent) with hydrolyzing time, but peak 1 and 5 notably increased with time, peak 3 exhibited first increased and then decreased trend in hydrolyzate during the hydrolysis. At end of $\mathrm{HCl}$ hydrolysis (300 min), a small proportion of oligosaccharides, indicated with peaks 1-3, 5, are yet observed on HPLC profile. Semi-quantification for the relative content of each oligomer in $\mathrm{HCl}$ hydrolyzate was expressed with area percentage of every peak accounting for the total peaks, and its change with hydrolyzing time can be seen in Fig. 3. It is shown that treatment of MVPS with $\mathrm{HCl}$ can produce various hydrolyzates, whose oligosaccharides composition and compounds content were largely depended on time control.

\subsection{Oligosaccharides identification of the MVPS hydrolyzate}

HPLC chromatography coupled with mass spectrometry is a very powerful analytical technique, due to its high sensitivity, and the structural information that can be obtained about the analytes (Vismeh, Humpula, Chundawat, Balan, Dale, \& Jones, 2013; Wang, Yuan, Sun, Cao, Tian, \& Wang, 2009). In this study, HPLC-ESI-TOF-MS was used to directly identify the oligomers in MVPS hydrolyzate. The hydrolyzate produced by $1 \mathrm{M} \mathrm{HCl}$ hydrolysis for $1 \mathrm{~h}$ was selected as representive analytes for HPLC-ESI-TOF-MS analysis, because it contains abundant producing oligosaccharides and show total characteristic compound peaks in HPLC-ELSD profile. The result of total ion chromatogram at positive model $\left([\mathrm{M}+\mathrm{Na}]^{+}\right)$for oligosaccharides in MVPS hydrolyzate eluted from $\mathrm{NH}_{2} \mathrm{P}-4 \mathrm{E}$ (NP) column by HPLC separation was shown in Fig. 4a, which combined with extracted molecules mass ion chromatograms for all oligosaccharides (Fig. 4b, c, d, e, f, g, h). In the total ion chromatogram (Fig. 4a), nine compound structures are co-eluted from $\mathrm{NH}_{2} \mathrm{P}-4 \mathrm{E}$ column in the periods of 9.58-28.00 min, the relative retention time of the major populations agree well with those obtained from HPLC profiles, thus were labeled with same number signals (1-9). These nine ion peaks were respectively extracted with molecule mass from the total ion chromatogram. Extracted ion of compound 1 eluted at $10.58 \mathrm{~min}$ in LC-MS chromatogram shows the $[\mathrm{M}+\mathrm{Na}]^{+},[2 \mathrm{M}+\mathrm{Na}]^{+}$and $[3 \mathrm{M}+\mathrm{Na}]^{+}$adduct ions peaks with $\mathrm{m} / \mathrm{z}=203.06,707.29$ and 1071.43 
respectively, indicating that its molecule mass is $180 \mathrm{Da}$ and recognized as $\alpha$-D-glucose. The appearance of polymolecule ion peaks might be attributed to the higher concentration of compounds in analyte, which was usually reported in other research (Hsu, Chang, \& Franz, 2006). Oligosaccharides 2 and 3 that co-eluted at 14.09 and $15.13 \mathrm{~min}$ are two dimers of glucose with same molecule mass $342 \mathrm{Da}$ because they were found having $[\mathrm{M}+\mathrm{Na}]^{+}$adduct ions with $\mathrm{m} / \mathrm{z}=$ 365.13 in MS spectra. Similarly, Oligosaccharides 4 and 5 co-eluted at 17.34 and 18.14 min are two trimers of glucose with same molecule mass $504 \mathrm{Da}\left(\mathrm{m} / \mathrm{z}\right.$ of $[\mathrm{M}+\mathrm{Na}]^{+}$are 527.19 and 527.20). The existence of these geometric isomers was caused by two different glycosidic linkages between

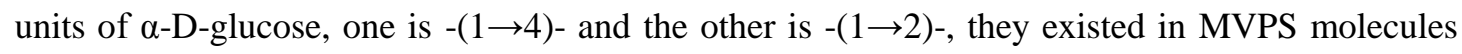
with 6:1 of mole ratio (Wang, Zhang, Di, Liu, \& Wu, 2011). As the hydrolyzing products of MVPS, the oligosaccharides 2 and 3 are here identified as Glu- $\alpha-(1 \rightarrow 4)$-Glu dimer (Maltose) and Glu- $\alpha-(1 \rightarrow 2)$-Glu dimer (Kojibiose), respectively. The oligosaccharides 4 and 5 with DP=3 are hence confirmed as Glu- $\alpha-(1 \rightarrow 4)$-Glu- $\alpha-(1 \rightarrow 4)$-Glu trimer (Maltotriose) and Glu- $\alpha-(1 \rightarrow 4)$-Glu- $\alpha-(1 \rightarrow 2)$-Glu trimer, respectively. Their completely chemical structures were drawn on the corresponding MS spectra and shown in the Fig. 5. Accordingly, via recognition of the molecules ion by ESI-TOF-MS analysis, the adduct ion peaks eluted at 20.18, 22.48, 24.12 and 25.53 min were identified as glucooligosaccharides with $\mathrm{DP}=4,5,6$ and 7, respectively (Fig. 6). All these glucooligosaccharides are still confirmed as isomer mixtures, containing two or more types of molecules due to the co-existence of two types of glycosidic linkage (e.g., Glu- $\alpha-(1 \rightarrow 2)$-Glu structures with $\left.[\alpha-(1 \rightarrow 4)-G l u]_{n}\right)$. The positional isomers of oligosaccharide with higher DP $(\geq 4)$ are very difficult to distinguish by TOF-MS analysis because the NP-HPLC profiling was unable to separate them. This limitation resulted in the shouldering peak appearance for the higher DP oligosaccharides in the profile. Thus complicated fragment ions presented on the MS spectrums for some higher DP of oligosaccharides and caused the problem in precisely elucidating. Considering this, the peaks eluted from HPLC profile at different retention time in the range of 8-28 min was confirmed as homogeneous oligosaccharides and thereby expressed with various $\mathrm{DP}(\mathrm{DP}=1$ for peak1, $\mathrm{DP}=2$ for peaks 2 and 3, $\mathrm{DP}=3$ for peaks 4 and $5, \mathrm{DP}=4$ for peak 6 , $\mathrm{DP}=5$ for peak $7, \mathrm{DP}=6$ for peak 8 , and $\mathrm{DP}=7$ for peak 9 ). All of them are rational products of MVPS under the mild acid hydrolysis.

\subsection{Antioxidant activities}




\subsubsection{DPPH radical-scavenging activity}

DPPH is a useful reagent for investigating the free radical-scavenging activities of materials. Antioxidants transfer either electrons or hydrogen atoms to DPPH and thus neutralize free radical (Naik et al., 2003). It is noticeable by eye that there is a discolouration from purple to yellow induced by antioxidants. Fig. 7a illustrated the results on scavenging activity of the tested samples to DPPH radicals. It suggested that all samples had inhibitory effects on DPPH radical in a concentration-dependent manner from 10 to $2000 \mu \mathrm{g} / \mathrm{mL}$. Among the three tested samples, $\mathrm{HCl}$ hydrolyzate has the strongest ability in DPPH radical-scavenging and its concentration inhibiting $50 \%$ radical generation $\left(\mathrm{IC}_{50}\right)$ is $482 \mu \mathrm{g} / \mathrm{mL}$. MVPS has a moderate DPPH radical-scavenging ability with $\mathrm{IC}_{50}=709 \mu \mathrm{g} / \mathrm{mL}$. However, inhibitory effect of $\mathrm{H}_{2} \mathrm{SO}_{4}$ hydrolyzate is weakest with $\mathrm{IC}_{50}=1246 \mu \mathrm{g} / \mathrm{mL}$ and just $69 \%$ of inhibitory can be reached even at as high as $2000 \mu \mathrm{g} / \mathrm{mL}$ of concentration. The difference in ability to quench DPPH free radical among $\mathrm{HCl}$ hydrolyzate, MVPS and $\mathrm{H}_{2} \mathrm{SO}_{4}$ hydrolyzate might be caused by amount of some special oligosaccharide contains. Those oligosaccharides might have stronger activities than the long chain polysaccharide and monose forms, which have been confirmed by many reports (Sun, Wang, Shi, \& Ma, 2009; $\mathrm{Wu}$, \& $\mathrm{Yu}, 2015)$. The $\mathrm{H}_{2} \mathrm{SO}_{4}$ hydrolyzate contains fewer long chain molecules and oligosaccharides, thus have the lowest DPPH scavenging activity.

\subsubsection{Inhibitory effects on hydroxyl radical}

Comparing with other reactive oxygen species, hydroxyl radical has the highest activity which could induce severe damage to organism. Previous studies had reported that suppression against hydroxyl radical generation and cleaning the hydroxyl radical generated might be responsible for the antioxidative effects of natural polysaccharides (Shon, Kim, \& Sung, 2003, Xing, Liu, Yu, Zhang, Li, \& Li, 2004). The EDTANa2-Fe (II)- $\mathrm{H}_{2} \mathrm{O}_{2}$ system was used to generate hydroxyl radical $(\bullet \mathrm{OH})$ which would discolor safranin T. Added hydroxyl radical inhibitor could inhibit the bleaching. The above mentioned model was used to measure inhibitory effects of the three tested samples on hydroxyl radical and compared with that of Vc. The results indicated that inhibitory effects of the samples were concentration-dependent in the range of $10-2000 \mu \mathrm{g} / \mathrm{mL}$ and samples with higher concentration induce better effect (see Fig. 7b). The $\mathrm{IC}_{50}$ of MVPS, HCl hydrolyzate and $\mathrm{H}_{2} \mathrm{SO}_{4}$ hydrolyzate was 309,485 , and $1489 \mu \mathrm{g} / \mathrm{mL}$ respectively. Inhibitory of vitamin $\mathrm{C}$ to hydroxyl radical are obviously higher than the three test samples, which was between 
$80 \%$ and $90 \%$ at $100 \mu \mathrm{g} / \mathrm{mL}$ and accorded with the previous reports ( $\mathrm{Li}$ et al., 2013). The results also showed the inhibitory effects of $\mathrm{HCl}$ hydrolyzate on hydroxyl radical were keeping on higher levels than MVPS and $\mathrm{H}_{2} \mathrm{SO}_{4}$ hydrolyzate. It was suggested that containing more oligosaccharides would enhance the hydroxyl radical inhibitory effect, because sugar contents were almost equal between $\mathrm{HCl}$ and $\mathrm{H}_{2} \mathrm{SO}_{4}$ hydrolyzates, However, the antioxidants of the test samples were not depended on single factor but a combination of several factors, varying from contents composition to spatial structure and groups (Song, Zhang, Zhang, \& Wang, 2010).

\subsubsection{Reducing power}

In the reaction system, antioxidant substances in samples cause reduction of $\mathrm{Fe}^{3+} /$ ferricyanide complex to the $\mathrm{Fe}^{2+}$ form. Therefore, $\mathrm{Fe}^{2+}$ can be monitored by measuring the formation of Prussian blue at $700 \mathrm{~nm}$. Higher absorbance value means stronger reducing power of sample. Fig. 7c depicts the reducing powers of the tested samples that exhibited concentration-dependent increasing manner. At $400 \mu \mathrm{g} / \mathrm{mL}$, the reducing powers were $0.23,0.20$, and 0.11 for MVPS, $\mathrm{HCl}$ hydrolyzate, and $\mathrm{H}_{2} \mathrm{SO}_{4}$ hydrolyzate, respectively. At $1200 \mu \mathrm{g} / \mathrm{mL}$, the reducing powers were 0.89 , 0.95, and 0.58 for MVPS, $\mathrm{HCl}$ hydrolyzate, and $\mathrm{H}_{2} \mathrm{SO}_{4}$ hydrolyzate, respectively. $\mathrm{HCl}$ hydrolyzate showed slightly higher activity than that of the other two samples. The reducing power was generally associated with the presence of reductones in oligosaccharides and polysaccharides, which had been shown to exert antioxidant action by breaking the free radical chain by donating a hydrogen atom. Reductones were also reported to react with certain precursors of peroxide, thus preventing peroxide formation (Li et al., 2013; Song, Zhang, Zhang, \& Wang, 2010). The results suggest that all the samples might supply reducing agents or groups to enhance their antioxidant activities.

\section{Conclusion}

This study investigated the degradable characteristics of water soluble polysaccharides from M. veneriformis (MVPS) with controlled acid hydrolysis method for the first time. Results confirmed that MVPS can easily be hydrolyzed by two types of mineral acids, $\mathrm{HCl}$ and $\mathrm{H}_{2} \mathrm{SO}_{4}$, producing some MVPS fragments formed with oligosaccharides and glucose in hydrolyzate. The hydrolyzing reaction was investigated in three aspects, type of acid, acid addition amount and reaction time. The acid type and addition amount influence the hydrolyzing rate of MVPS. $\mathrm{H}_{2} \mathrm{SO}_{4}$ 
usage in hydrolysis can rapidly induce the MVPS degrading to monose, while $\mathrm{HCl}$ processing to MVPS behave a time-depended hydrolyzing manner, which is mild and controllable. During the acidic processing, high molecule weight of MVPS will be gradually hydrolyzed to low molecule weight of oligomers of polysaccharide. The change in oligomers compositions and relatively contents for carbohydrate profile of hydrolyzate with time is detailed by HPLC method. Total nine eluting peaks are recognizable in the carbohydrate profile of MVPS hydrolyzates, and then they are identified as glucooligosaccharides with DP from 1 to 7 by HPLC-ESI-TOF-MS analysis.

Representative hydrolyzates by $\mathrm{HCl}$ and $\mathrm{H}_{2} \mathrm{SO}_{4}$ processing were further investigated their antioxidant activities, comparing with the initial polysaccharide (MVPS) and positive control (Vc). In brief, initial polysaccharide and the polysaccharide hydrolyzates with different oligosaccharides contents all possessed antioxidant activities, performing with scavenging DPPH radicals, inhibiting the hydroxyl radicals and generating reducing power at relative low concentrations, and sample marked with $\mathrm{HCl}$ hydrolyzate exhibited stronger antioxidant effects. The study indicated the oligosaccharides content might be a significant factor to the antioxidant activity when sugar content and monosaccharide composition of the samples were similar. However, the mechanism of antioxidant activities in vivo and the effectiveness of $\mathrm{HCl}$ hydrolyzate from MVPS for human consumption should be further investigated.

\section{Acknowledgements}

The financial grants of this work have been co-supported by A Project Funded by the Priority Academic Program Development of Jiangsu Higher Education Institutions (PAPD), the National Science Foundation of China (NSFC, No. 30900293) and the commonweal items of State Oceanic Administration People's Republic of China (No. 201205022-8, No. 201305007 and No. 201405017).

\section{Reference}

Aam, B. B., Heggset, E. B., Norberg, A. L., Sorlie, M., Varum, K. M., \& Eijsink, V. G. H. (2010). Production of Chitooligosaccharides and Their Potential Applications in Medicine. Marine Drugs, 8(5), 1482-1517.

Agbagla-Dohnani, A., Cornu, A., \& Broudiscou, L. P. (2012). Rumen digestion of rice straw structural polysaccharides: effect of ammonia treatment and lucerne extract supplementation in vitro. Animal, 6(10), $1642-1647$. 
Al Jamal, R., Roughley, P. J., \& Ludwig, M. S. (2001). Effect of glycosaminoglycan degradation on lung tissue viscoelasticity. American Journal of Physiology-Lung Cellular and Molecular Physiology, 280(2), L306-L315.

Allerdings, E., Ralph, J., Steinhart, H., \& Bunzel, M. (2006). Isolation and structural identification of complex feruloylated heteroxylan side-chains from maize bran. Phytochemistry, 67(12), 1276-1286.

Apolinario, A. C., Goulart de Lima Damasceno, B. P., de Macedo Beltrao, N. E., Pessoa, A., Converti, A., \& da Silva, J. A. (2014). Inulin-type fructans: A review on different aspects of biochemical and pharmaceutical technology. Carbohydrate Polymers, 101, 368-378.

Arrizon, J., Morel, S., Gschaedler, A., \& Monsan, P. (2010). Comparison of the water-soluble carbohydrate composition and fructan structures of Agave tequilana plants of different ages. Food Chemistry, 122(1), 123-130.

Avila-Fernandez, A., Galicia-Lagunas, N., Rodriguez-Alegria, M. E., Olvera, C., \& Lopez-Munguia, A. (2011). Production of functional oligosaccharides through limited acid hydrolysis of agave fructans. Food Chemistry, 129(2), 380-386.

Barroso, B., Dijkstra, R., Geerts, M., Lagerwerf, F., van Veelen, P., \& de Ru, A. (2002). On-line high-performance liquid chromatography/mass spectrometric characterization of native oligosaccharides from glycoproteins. Rapid Communications in Mass Spectrometry, 16(13), 1320-1329.

Beveridge, R. J., \& Richards, G. N. (1973). Digestion of polysaccharide constituents of tropical pasture herbage in the bovine rumen. IV. The hydrolysis of hemicelluloses from spear grass by cell-free enzyme systems from rumen fluid. Carbohydrate research, 29(1), 79-87.

Braca, A., De Tommasi, N., Di Bari, L., Pizza, C., Politi, M., \& Morelli, I. (2001). Antioxidant principles from Bauhinia tarapotensis. Journal of Natural Products, 64(7), 892-895.

Chen, H. M., Zheng, L., Lin, W., \& Yan, X. J. (2004). Product monitoring and quantitation of oligosaccharides composition in agar hydrolysates by precolumn labeling HPLC. Talanta, 64(3), 773-777.

de Moura, F. A., Macagnan, F. T., \& da Silva, L. P. (2015). Oligosaccharide production by hydrolysis of polysaccharides: a review. International Journal of Food Science and Technology, 50(2), 275-281.

Du, B., Song, Y., Hu, X., Liao, X., Ni, Y., \& Li, Q. (2011). Oligosaccharides prepared by acid hydrolysis of polysaccharides from pumpkin (Cucurbita moschata) pulp and their prebiotic activities. International Journal of Food Science and Technology, 46(5), 982-987.

Hsu, J., Chang, S. J., \& Franz, A. H. (2006). MALDI-TOF and ESI-MS analysis of oligosaccharides labeled with a new multifunctional oligosaccharide tag. Journal of the American Society for Mass Spectrometry, 17(2), 194-204.

Juskiewicz, J., Zdunczyk, Z., Jankowski, J., Krol, B., \& Milala, J. (2008). Gastrointestinal tract metabolism of young turkeys fed diets supplemented with pure nystose or a fructooligosaccharide mixture. Archives of Animal Nutrition, 62(5), 389-403.

Kai, Z., Pengju, X. U. E., \& Guangye, G. U. (2008). Study on Determination of Reducing Sugar Content Using 3,5-Dinitrosalicylic Acid Method. Food Science, 29(8), 534-536.

Kasai, A., Horie, H., \& Sakamoto, W. (2004). Selection of food sources by Ruditapes philippinarum and Mactra veneriformis (Bivalva : Mollusca) determined from stable isotope analysis. Fisheries Science, 70(1), 11-20.

Laurentin, A., \& Edwards, C. A. (2003). A microtiter modification of the anthrone-sulfuric acid colorimetric assay for glucose-based carbohydrates. Analytical Biochemistry, 315(1), 143-145.

Li, B., Liu, S., Xing, R., Li, K. C., Li, R. F., Qin, Y. K., et al., (2013). Degradation of sulfated polysaccharides from Enteromorpha prolifera and their antioxidant activities. Carbohydrate Polymers, 92(2), 1991-1996.

Luan, H.-M., Wang, L.-C., Wu, H., Jin, Y., \& Ji, J. (2011). Antioxidant activities and antioxidative components in the surf clam, Mactra veneriformis. Natural Product Research, 25(19), 1838-1848. 
Naik, G. H., Priyadarsini, K. I., Satav, J. G., Banavalikar, M. M., Sohoni, D. P., Biyani, M. K., et al., (2003). Comparative antioxidant activity of individual herbal components used in Ayurvedic medicine. Phytochemistry, 63(1), 97-104.

Shon, M. Y., Kim, T. H., \& Sung, N. J. (2003). Antioxidants and free radical scavenging activity of Phellinus baumii (Phellinus of Hymenochaetaceae) extracts. Food Chemistry, 82(4), 593-597.

Sun, L. Q., Wang, C., Shi, Q. J., \& Ma, C. H. (2009). Preparation of different molecular weight polysaccharides from Porphyridium cruentum and their antioxidant activities. International Journal of Biological Macromolecules, $45(1), 42-47$.

Vismeh, R., Humpula, J. F., Chundawat, S. P. S., Balan, V., Dale, B. E., \& Jones, A. D. (2013). Profiling of soluble neutral oligosaccharides from treated biomass using solid phase extraction and LC-TOF MS. Carbohydrate Polymers, 94(2), 791-799.

Wang, J., Yuan, X., Sun, B., Cao, Y., Tian, Y., \& Wang, C. (2009). On-line separation and structural characterisation of feruloylated oligosaccharides from wheat bran using HPLC-ESI-MSn. Food Chemistry, 115(4), $1529-1541$.

Wang, L. C., Di, L. Q., Liu, R., \& Wu, H. (2013). Characterizations and microsphere formulation of polysaccharide from the marine clam (Mactra veneriformis). Carbohydrate Polymers, 92(1), 106-113.

Wang, L. C., Zhang, K., Di, L. Q., Liu, R., \& Wu, H. (2011). Isolation and structural elucidation of novel homogenous polysaccharide from Mactra veneriformis. Carbohydrate Polymers, 86(2), 982-987.

Wang L. C., Wu H., Chang N., \& Zhang K. (2011). Anti-hyperglycemic effect of the polysaccharide fraction isolated from Mactra veneriformis, Frontier of Chemical Enginerring In China, 5(2):238-244

Wu, S. J., \& Yu, L. (2015). Preparation and characterisation of the oligosaccharides derived from Chinese water chestnut polysaccharides. Food Chemistry, 181, 15-18.

Xing, R. E., Liu, S., Yu, H. H., Zhang, Q. B., Li, Z., \& Li, P. C. (2004). Preparation of low-molecular-weight and high-sulfate-content chitosans under microwave radiation and their potential antioxidant activity in vitro. Carbohydrate Research, 339(15), 2515-2519.

Yuan, Y. V., Carrington, M. F., \& Walsh, N. A. (2005). Extracts from dulse (Palmaria palmata) are effective antioxidants and inhibitors of cell proliferation in vitro. Food and Chemical Toxicology, 43(7), 1073-1081.

Zheng, W. W., Wang, L. C., Wu, H., \& Luan, H. M. (2011). Immune-Modulation of Crude Polysaccharide from Mactra Veneriformis. China Pharmacist, 14(2), 151-153.

Song, H., Zhang, Q., Zhang, Z., \& Wang, J. (2010). In vitro antioxidant activity of polysaccharides extracted from Bryopsis plumosa. Carbohydrate Polymers, 80(4),1057 - 1061. 


\section{Figure Caption:}

Fig. 1 The hydrolyzing isotherms of MVPS under the hydrolysis of $\mathrm{HCl}$ (a) and $\mathrm{H}_{2} \mathrm{SO}_{4}$ (b). The reaction was carried out with $10 \%$ (w/v) MVPS in 0.1, 1.0, 2.0 and 3.0 $\mathrm{M}$ acid at $60{ }^{\circ} \mathrm{C}$. The degree of hydrolysis is expressed as GE and read as \% (see Section 2.3 for definition).

Fig. 2 The evolution on HPLC-ELSD profiles of oligosaccharides in hydrolyzates of 1.0 $\mathrm{M} \mathrm{HCl}(\mathrm{A})$ and $\mathrm{H}_{2} \mathrm{SO}_{4}$ (B) hydrolyzing for different time. Profiles (a), (b), (c), (d), (e), (f), (g) and (h) are the hydrolyzate samples that disposed with acids hydrolysis for $30,60,120,180,240,300$, and 360 min, respectively. Peaks 1-9 are denoted as oligosaccharides in hydrolyzate of MVPS.

Fig. 3 The relative content (peak areas) of the recognizable oligosaccharides from MVPS hydrolyzate at various disposal time of $1.0 \mathrm{M} \mathrm{HCl}$.

Fig. 4 The total ionization chromatography (a) and extracted ion chromatograms $\left([\mathrm{M}+\mathrm{Na}]^{+}\right)$for various glucose oligomers in $\mathrm{HCl}$ hydrolyzate. (b) Extracted ion at $\mathrm{m} / \mathrm{z}$ $=203.05$ for peak 1 ; (c) extracted ion at $\mathrm{m} / \mathrm{z}=365.15$ for peaks 2 and 3 ; (d) extracted ion at $\mathrm{m} / \mathrm{z}=517.20$ for peaks 4 and 5 ; (e) extracted ion at $\mathrm{m} / \mathrm{z}=689.29$ for peak 6 ; (f) extracted ion at $\mathrm{m} / \mathrm{z}=851.37$ for peak 7 ; $(\mathrm{g})$ extracted ion at $\mathrm{m} / \mathrm{z}=1013.44$ for peak 8 ; (h) extracted ion at $\mathrm{m} / \mathrm{z}=1175.49$ for peak 9 .

Fig. 5 Structure elucidation and ESI-TOF-MS spectra (in positive mode) of the oligosaccharides in $\mathrm{HCl}$ hydrolyzate that retained at HPLC column from 10.58 to 18.15 min. MS spectra of (a), (b), (c), (d), and (e) are of the compound peaks of 1, 2, 3, 4 , and 5 , respectively.

Fig. 6 Structure elucidation and ESI-TOF-MS spectra (in positive mode) of the oligosaccharides in $\mathrm{HCl}$ hydrolyzate that retained at HPLC column from 20.18 to 25.13 min. MS spectra of (a), (b), (c), and (d) are of the compound peaks of 6, 7, 8, and 9 , respectively.

Fig. 7 The DPPH radicals scavenging (a), hydroxyl radicals scavenging (b), and reducing power (c) for MVPS, and its $\mathrm{HCl}$ and $\mathrm{H}_{2} \mathrm{SO}_{4}$ hydrolyzates. $\mathrm{Vc}$ as the positive control. Each sample was assayed in triplicate for each concentration. ${ }^{*} P<$ 0.05 and $* * P<0.001$ vs. group of $\mathrm{H}_{2} \mathrm{SO}_{4}$ hydrolyzate. 

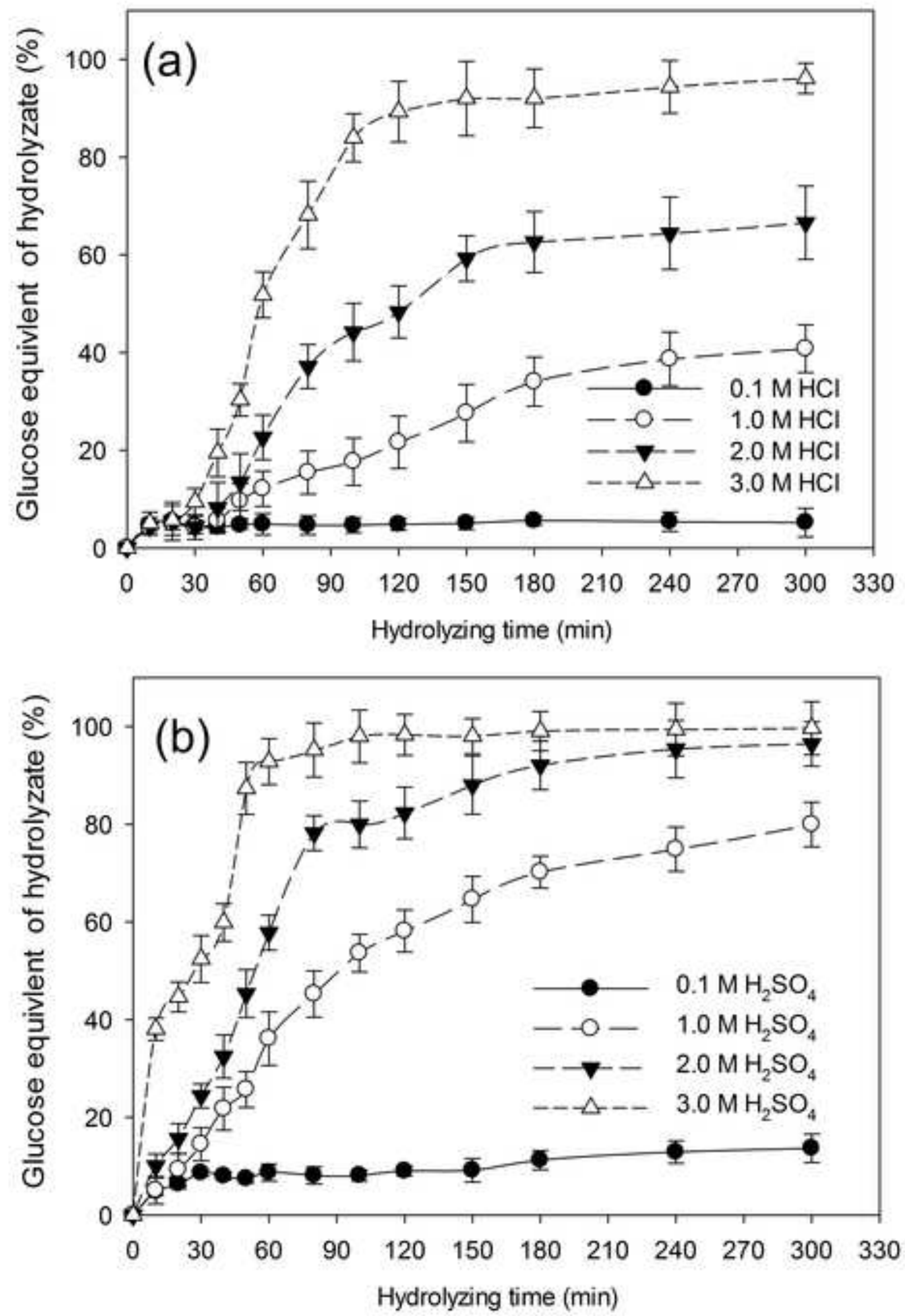

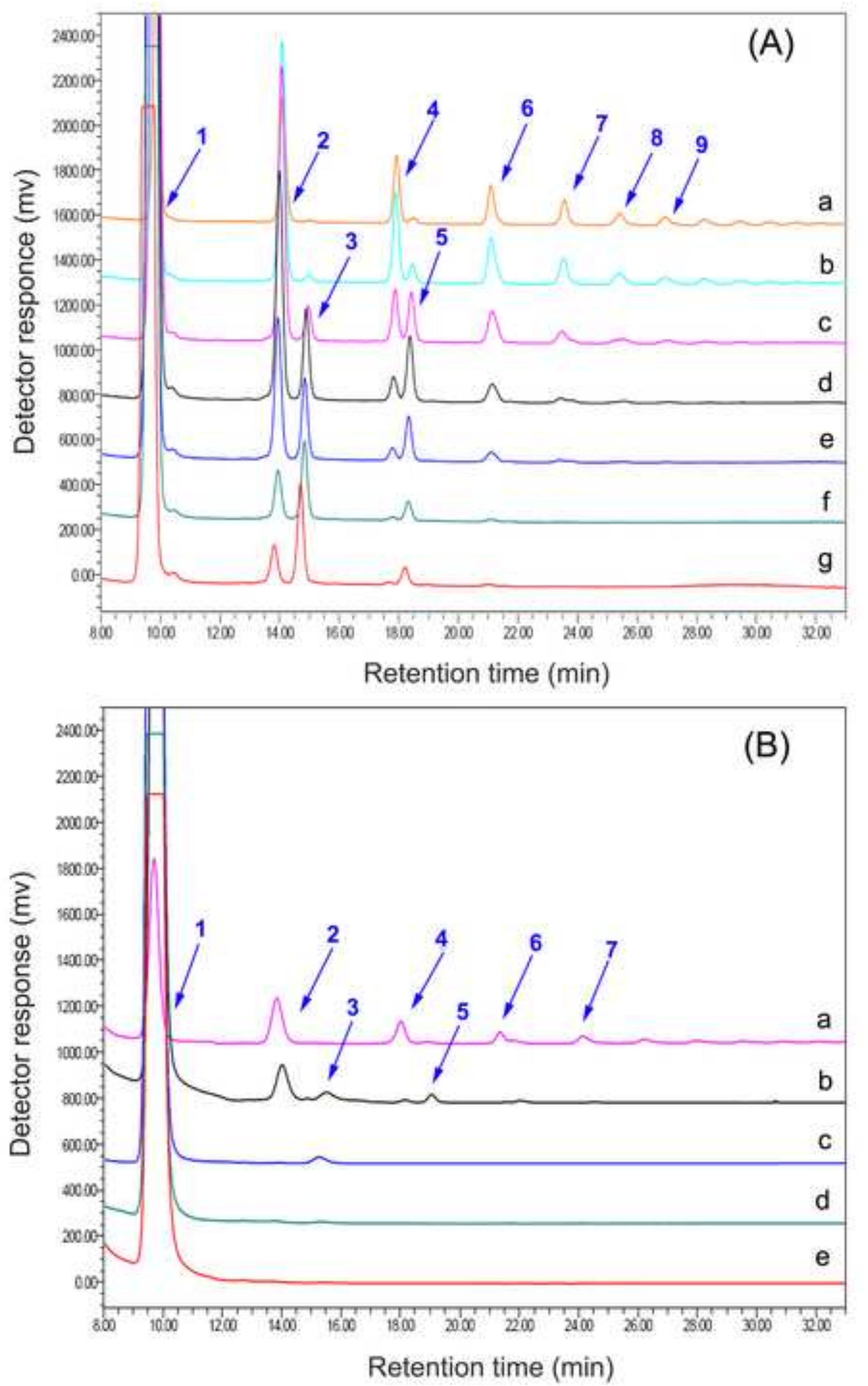

Figure 2

.

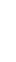

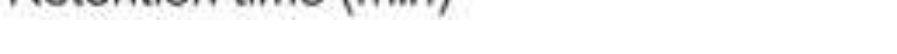

Figure

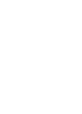




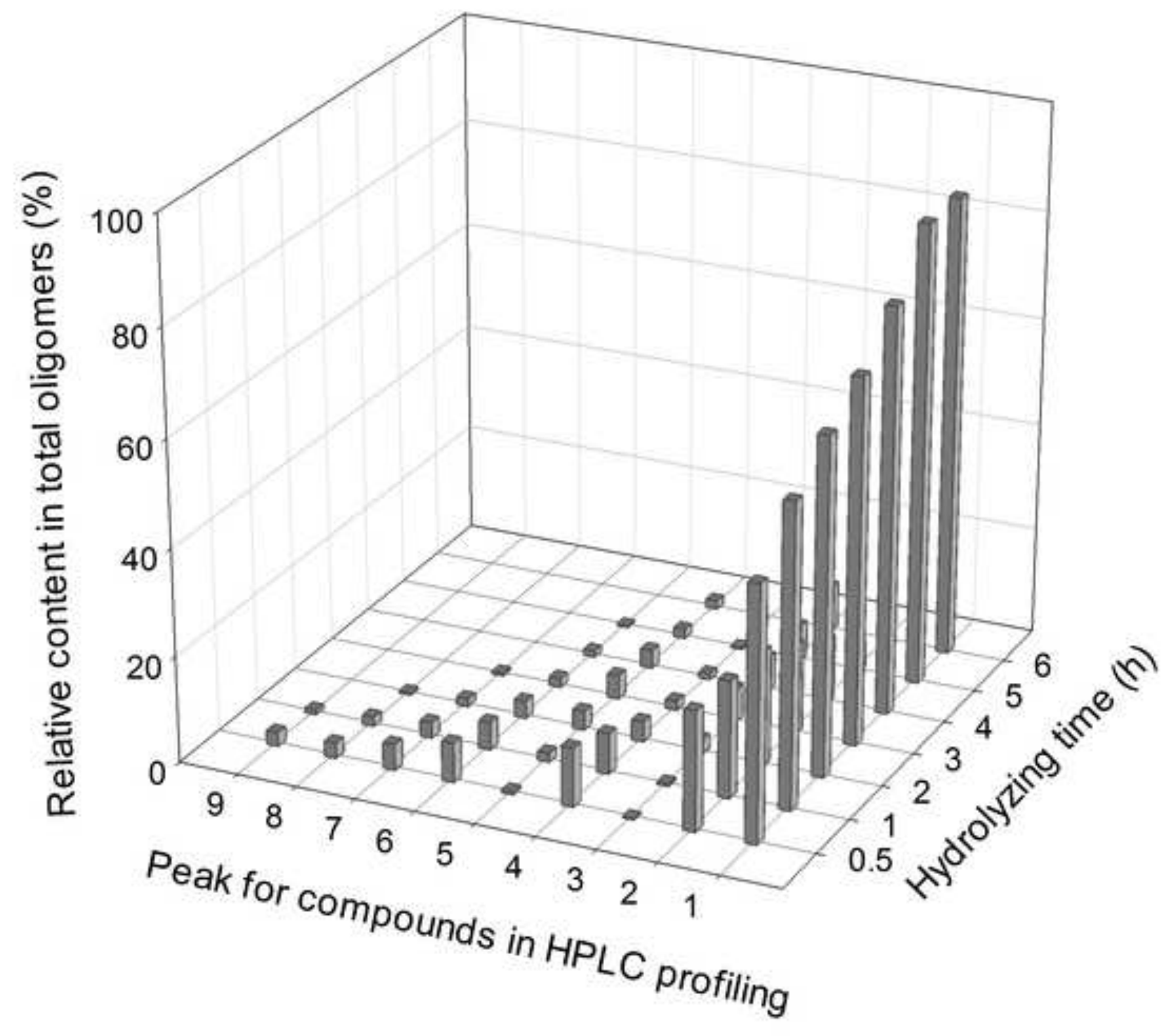



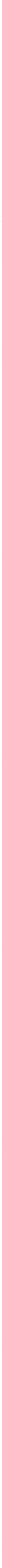
OFigure 5 ride 1 at $10.58 \mathrm{~min}$

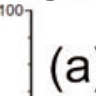

$\mathrm{m} / \mathrm{z}=203.0589$

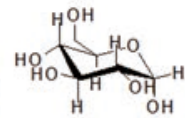

383.1490 $[2 \mathrm{M}+\mathrm{Na}]^{+}$

1: TOF MS ES+

$\mathrm{m} / \mathrm{z}=383.1490$

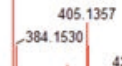

$\begin{array}{ll}421.1086 & 4430644.4510424 \quad 495.1236\end{array}$

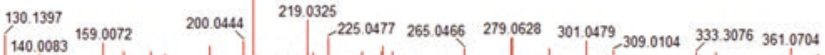

$[\mathrm{M}+\mathrm{Na}]^{+}$

1: TOF MS ES+

${ }^{100}$ (b)

365. 1352

$\mathrm{m} / \mathrm{z}=365.1532$

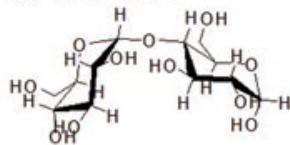

$[2 \mathrm{M}+\mathrm{Na}]^{+}$

2

70729732885

$362.1285 \int^{366.1432} 433.1335 \quad 533.2152 \quad 603.2062$

$\mathrm{m} / \mathrm{z}=707.2973$

$[3 \mathrm{M}+\mathrm{Na}]^{+}$

$\mathrm{m} / \mathrm{z}=1071.4316$

267.0610

$503.2152-603.2062$

Oligosaccharide 3 at $15.09 \mathrm{~min}$

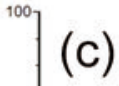

(c)

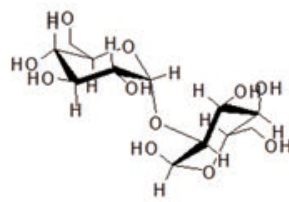

65.1352

$[\mathrm{M}+\mathrm{Na}]^{+}$

1: TOF MS ES+

$\mathrm{m} / \mathrm{z}=365.1352$

2e

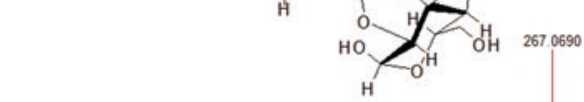

113.1088130 .1341

\begin{tabular}{ll|lll}
190.2197 & 261.1353 & 268.0750 & 297.0804 & 321.0791 .329 .0436
\end{tabular}

403.0554 Oligosaccharide 4 at $17.34 \mathrm{~min}$

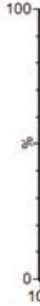

(d)

163.0642212 .2020

325.1504

$4 2 3 . 1 1 4 4 \quad 5 2 4 . 1 9 1 2 \longdiv { 5 2 9 . 2 2 1 3 } \quad 5 8 9 . 1 7 7 9$

$[\mathrm{M}+\mathrm{Na}]^{+}$

1: TOF MS ES+

Oligosaccharide 5 at $18.14 \mathrm{~min}$

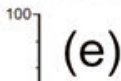

(e)

527.2019

$\mathrm{m} / \mathrm{z}=527.1906$

$[2 \mathrm{M}+\mathrm{Na}]^{+}$

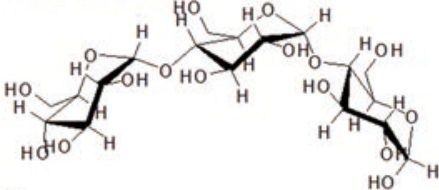

$[2 \mathrm{M}+\mathrm{Na}]^{+}$

$\mathrm{m} / \mathrm{z}=1031.4393$
$102.1357 \quad 2122020$

\begin{tabular}{l|lllll|}
167.0210 & 261.1511 & 288.9504 & 325.1591 & 429.1503 & 489.1784
\end{tabular}
328. 1998

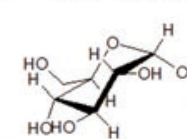

$[\mathrm{M}+\mathrm{Na}]^{+}$

1: TOF MS ES+

543.1918

bess.1698 101.1101

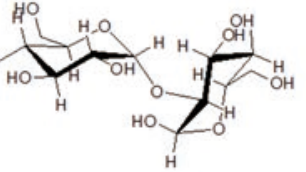

$1031,4393,1053,470$

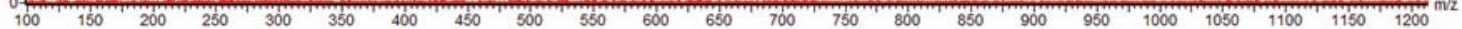


offigure 6 ide 6 eluted at $20.18 \mathrm{~min}$

(a)

$\mathrm{m} / \mathrm{z}=689.2904$

Glucooligosaccharides with $\mathrm{DP}=4$

Oligosaccharide 7 eluted at $22.48 \mathrm{~min}$

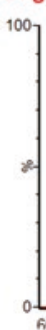

(b)<smiles>C1CCCCC1</smiles>

$[\mathrm{M}+\mathrm{Na}]^{+}$

1: TOF Ms ES+

Oligosaccharide 8 eluted at $24.12 \mathrm{~min}$

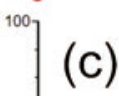

(c) $\mathrm{m} / \mathrm{z}=851.3722$

\section{Glucooligosaccharides with $\mathrm{DP}=5$}

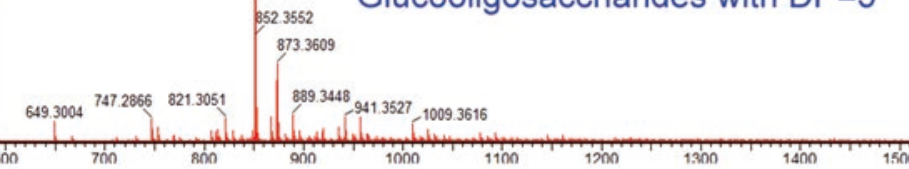

$[2 \mathrm{M}+\mathrm{Na}]^{+}$

$\mathrm{m} / \mathrm{z}=1679.3379$

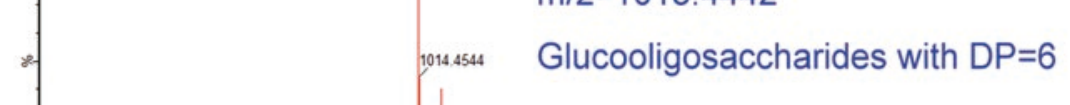

$$
\mathrm{m} / \mathrm{z}=1013.4442
$$

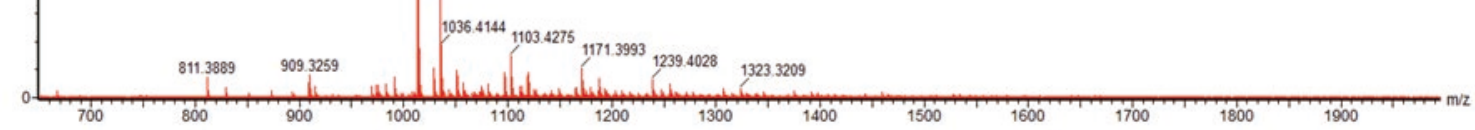

Oligosaccharide 9 eluted at $25.13 \mathrm{~min}$ 100

(d) 

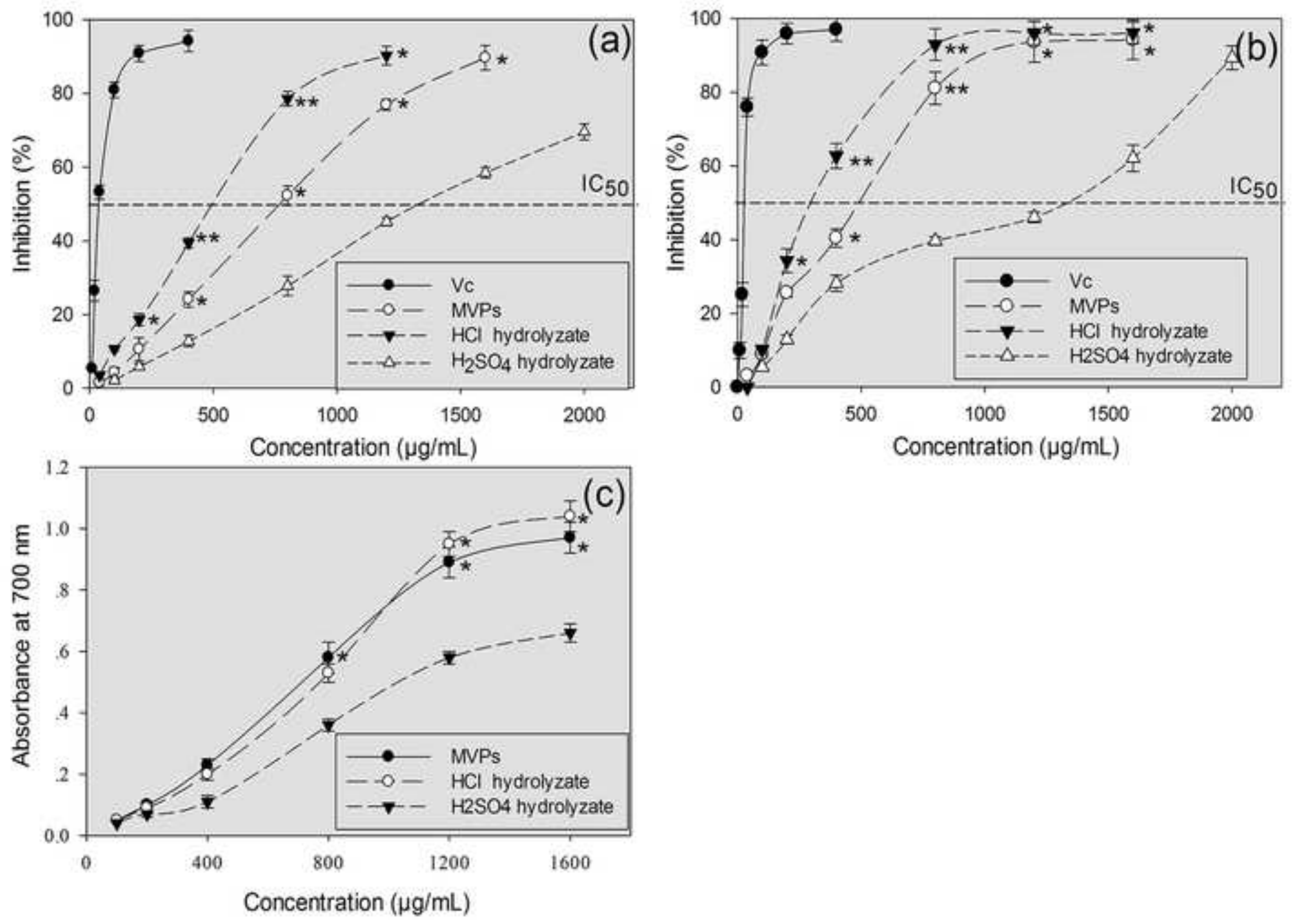